\title{
Biofuel alternatives to ethanol: pumping the microbial well
}

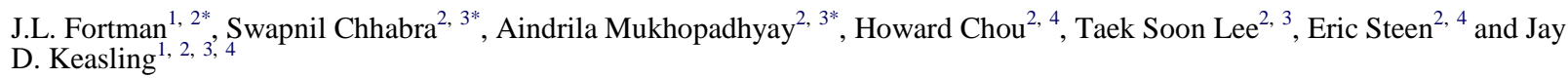 \\ ${ }^{1}$ Department of Chemical Engineering, University of California, 717 Potter Street, Building 977, Mail code 3224 \\ Berkeley, CA 94720-3224, USA \\ ${ }^{2}$ Joint BioEnergy Institute, Emeryville, CA 95608, USA \\ ${ }^{3}$ Physical Biosciences Division, Lawrence Berkeley National Laboratory, 717 Potter Street, Building 977, Mail code 3224 \\ Berkeley, CA 94720-3224, USA \\ ${ }^{4}$ Department of Bioengineering, University of California, 717 Potter Street, Building 977, Mail code 3224 Berkeley, CA $94720-$ \\ 3224, USA
}

Engineered microorganisms are currently used for the production of food products, pharmaceuticals, ethanol fuel and more. Even so, the enormous potential of this technology has yet to be fully exploited. The need for sustainable sources of transportation fuels has gener-ated a tremendous interest in technologies that enable biofuel production. Decades of work have produced a considerable knowledge-base for the physiology and pathway engineering of microbes, making microbial engineering an ideal strategy for producing biofuel. Although ethanol currently dominates the biofuel mar-ket, some of its inherent physical properties make it a less than ideal product. To highlight additional options, we review advances in microbial engineering for the production of other potential fuel molecules, using a variety of biosynthetic pathways.

\section{INTRODUCTION}

Conversion of biomass to biofuels has been the subject of intense research efforts since the 1970s. This work has recently gained significant political and scientific momen-tum owing to concerns about climate change, global energy security and petroleum supply. Global energy usage is projected to nearly double in the next two decades [1-3], and biological fuel production might serve as a sustainable, carbon-neutral energy source compatible with current engine technology. In an effort to offset increases in con-sumption and to limit the fossil fuel-related negative impacts on the environment, the US Department of Energy has established the goal of supplanting 30\% of gasoline consumption with cellulosic ethanol by 2030 [2]. Similarly, a European Union Directive (2003) aims to replace 5.75\% of all gasoline and diesel transport fuels with biofuels by 2010 [4].

Owing to physical and political limitations on arable land, it is believed that future biofuels will, by necessity, originate from abundantly available lignocellulosic bio-mass [5]. The liberation of monomeric sugars from this biomass is the subject of intensive research efforts that might be the key to the sustainability of any biofuel process. This extremely important area of research has been reviewed elsewhere [6,7]. Here, we concentrate on another important factor for the cost-effective production of biofuels from lignocellulosic feedstocks - namely the conversion of biomass hydrolysates (monosaccharides) to target molecules. We present some recent developments in microbial pathway modifications as a means of producing both currently used and prospective biofuels from agricul-turally prevalent sugars.

The use of microbial systems for the production of industrially relevant compounds has seen substantial gains in the past decade as a direct result of the genomics revolution. Further advances in gene regulation, protein engineering, pathway portability, synthetic biology and metabolic engineering will propel the development of cost-efficient systems for biofuel production. The current range of biofuels consists primarily of microbially derived ethanol and plant-based biodiesel ( Box 1). Although bio-diesel is favored in several European countries, ethanol dominates the majority of the world biofuel market, in-cluding that of the United States. The pros and cons associated with any potential biofuel present challenges and opportunities that are best addressed on a case-by-case basis, and these issues are therefore beyond the scope of this review. Instead, we cover advances in metabolic engineering to produce biomolecules that are currently being explored for the development as transportation fuels ( Figure 1).

Box 1. Current motor fuels and their biological counterparts

Gasoline is a complex mixture of hydrocarbons composed primarily of branched-chain alkanes and aromatics ranging from 4 to 12 carbons in length [78]. Similarly, petroleum diesel (No. 2) is a mixture of hydrocarbons ranging from 9 to 23 carbons in length, with an average length of 16 carbons. The types of hydrocarbons in gasoline and diesel have a strong impact on the properties of the fuel. For example, branching and unsaturation leads to greater octane numbers in gasoline [79] and lower cetane numbers in diesel [80]. Conversely, 
n-alkanes have higher cetane numbers and lower octane values. These differences highlight the need for multiple alternative fuels to supplant petroleum-based counterparts.

To date, research into gasoline substitutes has focused largely on ethanol. However, biosynthesis pathways in microbial systems, both natural and engineered, yield molecules that are similar or identical to those currently found in gasoline. These include straight- and branched-chain alkanes and alkenes from fatty acid and isoprenoid pathways, in addition to several alcohols and esters ( Figure 1). Establishing high titer-production of these molecules through the use of microbial cultures could serve to replace some or all of the stock currently coming from petroleum.

Currently, plant oils constitute the major biological source for diesel fuel alternatives (biodiesel). However, even the most efficient plant-based production is insufficient to meet current diesel usage levels without a dramatic increase in cultivation [81-83]. Research into the production of compounds from fatty acid pathways suggests that straight-chain alkanes, identical to those found in petroleum diesel, and fatty alcohols can also be derived from microbial sources. Although currently far from industrial applica-tion, the production of biodiesel by an engineered strain of E. coli [39] exemplifies an alternative means of producing fatty esters that has been enabled by microbial engineering. In addition to the hydrocarbons that constitute the bulk of transportation fuels, additives are used to improve the performance and stability of both gasoline and diesel fuel. Gasoline additives include anti-knock agents, anti-oxidants, lead scavengers, lubricants and detergents. Diesel fuel additives are generally used to eliminate the gelling of the fuel in cold weather. Two classes of commonly used additives or oxygenates are alcohols and ethers, both of which reduce emissions and replace the previously used toxic octane enhancers, such as benzene, and anti-knock agents such as lead.

In addition to ethanol, other biologically derived alcohols, such as butanol and isopentenol, can increase the octane rating of gasoline without significantly decreasing the energy density [42]. In the past, butanol was commercially produced through the use of microbial cultures, and isopentenol is currently accessible at the laboratory scale. Advances in bioengineering technologies might eventually provide an economically competitive means of producing these fuel additives.

\section{Pathway engineering}

\section{Sugar catabolism and the fermentation pathway}

Agriculturally derived lignocellulosic hydrolysates contain predominantly glucose and xylose and small amounts of arabinose, galacturonic acid and rhamnose, in addition to a large percentage of lignin residues (not addressed here). For the economical bioconversion of these feedstocks to commodity chemicals, efficient use of a high percentage of available monosaccharides is crucial. Although glucose metabolism is a nearly universal trait of living organisms, the ability to metabolize other sugars varies widely within microbes. Therefore, catabolism of monosaccharides has been an important target in the development of an optimized microbial platform. Pathways for xylose and arabinose use have recently been engineered into Saccharomyces cerevisiae [8-10] and Zymomonas mobilis [11]. Although developments in sugar catabolism were made in the context of homo-ethanol production, the advances are applicable to the production of a variety of fuel molecules through multiple biosynthetic pathways (discussed below).

Microbial production of ethanol has been reviewed extensively [12-14]. S. cerevisiae, the most commonly used organism for ethanol production, and Z. mobilis are known to be homo-ethanol fermenters, and wild-type Escherichia coli ferments sugars to a mixture of ethanol and organic acids (Figure 1). A vast amount of work has been done to engineer E. coli strains capable of homo-ethanol pro-duction [14]. By chromosomal integration of the pro-duction-of-ethanol (PET) operon from Z. mobilis, Ingram and coworkers [15] were able to engineer E. coli straincompression ignition engines and have consequently been developed for use as biodiesel. However, if biodiesel is to replace a significant portion of current petro-diesel needs, microbes might serve to provide a more consistent and scalable source for this commodity.

The variety of fatty acids available from microbial sources can potentially provide the mixture of chain lengths and branching required for an ideal fuel-blend ( Box 1). Microbial fatty acid biosynthesis and the diversity of naturally occurring fatty acids are well understood [25]. One high-yield source is the oleaginous algae Botryococcus braunii $[26,27]$. In this organism, up to $40 \%$ of dry cell weight consists of fatty acids. Different microalgal species have been shown to accumulate different amounts of lipid, ranging from 40-70\% of their biomass. Manipulating growth conditions such as $\mathrm{CO}_{2}$ supplementation, and nitro-gen and light limitation can increase the lipid content of these algae [28]. A more direct replacement of conventional diesel fuel might come from n-alkane production in bac-teria. This unusual trait has been reported to occur in Vibrio furnissii [29]. Available studies of alkane biosynthesis in this bacterium indicate that the enzymatic mechanism is a novel sequential reduction of $n$-hexadecanoic acid to n-hexadecane catalyzed by the enzyme FahR [29]. This work reported that the yield of the $\mathrm{C}_{16}$ alkane from the $\mathrm{C}_{16}$ fatty acid is greater than $70 \%$. However, a recent report disputes these findings [30]. Alkane production can also be facilitated by the decarbonylation of fatty aldehydes ( Figure 1) [31,32]. However, the rates of conversion of decarbonylases described to date are too slow for commercial application. 


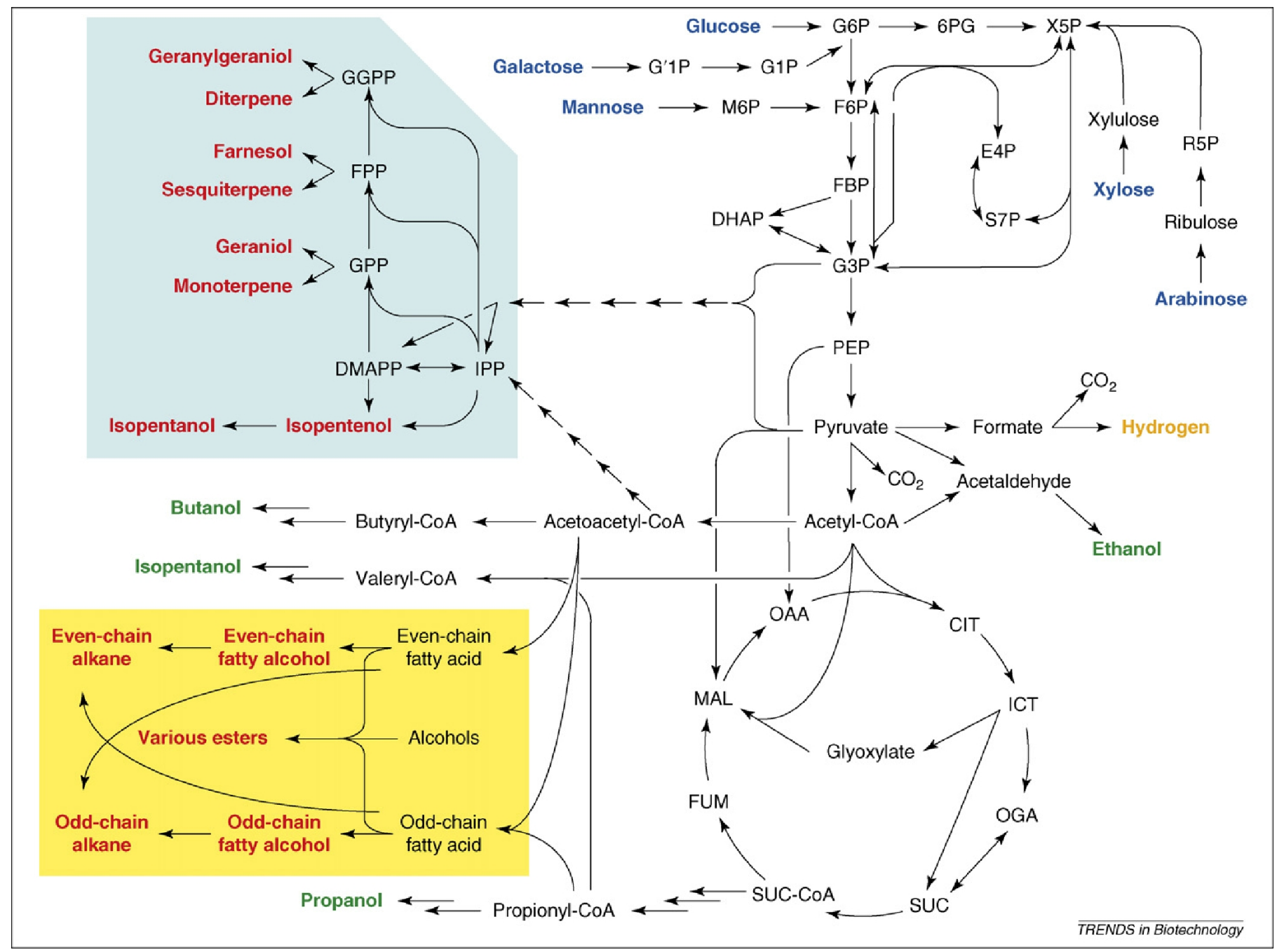

Figure 1. Central metabolic pathways and the potential fuel molecules derived from them. The blue box indicates isoprenoid pathways and isoprenoid-derived molecules. The yellow box indicates fatty acid pathways and fatty acid-derived molecules. Short-chain alcohols are shown in green text. Biomass-derived sugars are indicated in blue text. Abbreviations: 6P,G, 6-phosphogluconate; CIT, citrate; DHAP, dihydroxyacetone phosphate; DMAPP, dimethylallyl pyrophosphate; E4P, erythrose-4-phosphate; F6P, fructose-6-phosphate; FBP, fructose-1,6-bisphosphate; FPP, farnesyl pyrophosphate; FUM, fumarate; $\mathrm{G}^{0}$ 1P, galactose-1-phosphate; G1P, glucose-1-phosphate; G3P, glyceraldehyde-3-phosphate; G6P, glucose-6-phosphate; GGPP, geranylgeranyl pyrophosphate; GPP, geranyl pyrophosphate; ICT, isocitrate; IPP, isopentenyl pyrophosphate; M6P, mannose-6-phosphate; MAL, malate; OAA, oxaloacetate; OGA, 2-oxoglutarate; PEP, phosphoenolpyruvate; R5P, ribose-5-phosphate; S7P, sedoheptulose-7-phosphate; SUC, succinate; SUC-CoA, succinyl coenzyme A; X5P, xylulose-5-phosphate.

Fatty acid biosynthesis draws from the pool of acetyl-CoA produced by several central metabolic pathways. To improve the free fatty acid levels in microbes, the main approaches adopted to date have focused either on diverting the pool of acetyl-CoA towards fatty acid biosynthesis or on decreasing the cellular consumption of fatty acids. An example of the former method includes an engineered E. coli co-expressing several enzymes: an acetyl-CoA carboxy-lase, the first enzyme in the fatty acid pathway; a truncated thioesterase; and the fatty acid synthase. This leads to an increased production of free fatty acids [33]. Alternatively, to minimize fatty acid catabolism, genes such as yfcYX-ydiD in E. coli [34], and faa1, faa2 and faa3 in S. cerevisiae [35] have been targeted for deletion or inactivation. Also, deletion of the four genes associated with neutral lipid synthesis in S. cerevisiae (i.e. are1, are2, dga1 and lro1) results in a 2.5 -fold increase in the production of neutral fatty acids during late exponential and stationary growth phase [36].

Although fatty acids are the direct products of the fatty acid biosynthesis pathway, reduced and modified 
fatty compounds have also been made in bacteria. An example of microbial production of fatty alcohols and esters is the production of Jojoba oil-like wax esters and butyl esters in an engineered E. coli [37]. A recently isolated enzyme has also been shown to esterify fatty acids to isoprenyl alcohols, further expanding the range of possible bio-esters [38]. These fatty alcohols and esters can be produced with the appropriate chain length required to serve as biodiesel candidates ( Figure 1).

Biodiesel from microalgae-derived lipids has been explored extensively [26]. However, these studies still rely on a chemical transesterification to produce the fuel mol-ecules. Recently, Kalscheuer et al. [39] reported an E. coli-based process for in vivo transesterification. This FAEE biosynthesis was accomplished in recombinant E. coli by co-expression of the ethanol production genes from Z. mobilis in combination with the acyltransferase WS/ DGAT (wax ester synthase/acyl-coenzyme A:diacylglycerol acyltransferase) gene from Acinetobacter baylyi ADP1 [39]. Although this is an exciting proof of concept, the yields from this system need to be significantly improved for viable industrial application.

\section{The isoprenoid pathway}

The isoprenoids are a family of natural products that are synthesized with the use of the activated hydrocarbon monomers isoprenyl pyrophosphate (IPP) and its isomer dimethylallyl pyrophosphate (DMAPP) [40]. It is possible to produce several branched-chain alcohols, alkanes, alkenes and cyclic hydrocarbons through the isoprenoid biosynthetic pathway. For decades, this family of com-pounds has been posited as a potential source of biofuels [28,41]. In addition to branched-chain hydrocarbons, this pathway can be used to produce isopentanol (isoamyl alcohol) and its acetate ester, compounds proposed as additives for spark ignition fuels [42]. Sources of isopre-noids previously suggested for fuel production have included oil-producing algae such as B. braunii. These organisms produce large amounts of isoprenoids as well as fatty acids [27]. Owing to the complex mixture of hydro-carbons, the extract is more akin to a biocrude and there-fore would require hydrogenation and cracking, in much the same manner as fossil-derived counterparts [43]. Advances in the understanding and engineering of isopre-noid biosynthesis pathways might facilitate the production of either a similar biocrude or perhaps enginecompatible molecules from microorganisms.

Isoprenoid monomers are produced either from acetyl-CoA through the mevalonate pathway or from pyruvate and glyceraldehyde-3-phosphate (G3P) through the deoxyxylulose phosphate (DXP) (also known as the methy-lerythritol phosphate [MEP]) pathway $[40,44,45]$. These five-carbon monomers (i.e. IPP, DMAPP) can be polymer-ized to chains ranging in length from one to more than a dozen units [44,45]. Viable production of fuel molecules relies on both increasing precursor biosynthesis as well as optimizing several downstream steps.

The majority of the research in this area has come from studies of pharmaceutically important compounds such as lycopene, carotenoids, sterols, taxol and artemisinin [44,46-48]. High-level production of IPP has been achieved in both E. coli and S. cerevisiae, and many different iso-prenoids have been produced with the use of these engin-eered hosts [49-53]. In the engineering of E. coli hosts, the existence of two separate biosynthetic routes has been exploited by introducing the non-native mevalonate path-way. In yeast, the native mevalonate pathway has been improved by several approaches [50,54-56].

These advances in monomer production can be used to produce isoprenoid biofuels in an industrially tractable organism. Isoprenoids (e.g. phytoene [C40]) serve as inter-mediates in the production of large isoprenoid compounds, and they might be suitable as biocrude. Both E. coli and S. cerevisiae have been successfully engineered for the pro-duction of the phytoene-based carotenoids [51,57-59]; unfortunately, the current yields of these compounds fall far short of what is required if they are to produce viable fuel alternatives $[47,50,60]$. However, if sufficient titers of phytoene are reached, it could be refined into motor and jet fuel through the infrastructure currently used to process petroleum.

In addition to biocrude production, recent work suggests the isoprenoid pathway can be used to produce the pro-posed gasoline additives isopentanol and isoamylacetate [42]. For example, researchers have demonstrated that a pyrophosphatase isolated from Bacillus subtilis can depho-sphorylate IPP to form isopentenol [61]. The acetylation of isopentanol by an engineered E. coli has also been demon-strated [62,63]. Additionally, saturated, mono- or diunsa-turated monoterpenes and sesquiterpenes might be useful as diesel and jet fuels. The ability to modify terpene cyclases for the production of novel terpenes will greatly expand the number of potential fuels molecules that can be synthesized microbially [64-66].

\section{Major challenges and future directions}

Although known metabolic pathways offer several possible avenues for the biosynthesis of fuel molecules, several other factors need to be addressed before they can be applied in an industrial setting. First and foremost are the physical properties of the potential biofuel molecule. These properties have an impact on everything, from its 
suitability as a fuel to the purification processes and the mode of its transport to consumers. Although discussion of the properties for the biofuels mentioned herein is far beyond the scope of this review, this idea is nicely exemplified by the brief comparison of ethanol and butanol presented above.

The primary challenge in biofuel production is achieving yields that make these fuels cost-competitive with petroleum-based products. For any of the engineering approaches outlined in this review to be successful, the development of efficient lignocellulosic breakdown to monosaccharides is crucial. The development of this linchpin technology will enable the production of microbial-produced biofuels from crops that have higher growth-rates, better yields, lower soil-impacts, and lower water, fertilizer and pesticide requirements than currently used crops such as oil palms, corn or soybeans [67]. These characteristics should serve to make biofuels more com-petitive with petroleum, while making them less competi-tive with food production for arable land.

To date, pathway manipulations have largely been limited to the use of existing genes and conventional gene expression techniques. Although these approaches have yielded increases in target molecule production, a cellwide approach to metabolic engineering will be required to maximize the rate of biosynthesis in addition to the yield of the desired compound. To this end, there now exists a large body of work on the alteration of transcriptional and translational rates in addition to the development of evolved enzymes to maximize throughput. This knowledge is poised for use in the systematic design of highly efficient biofuel production pathways. This global approach is nicely illustrated by recent publications showing the effect of various transcription factors on ethanol tolerance and production in E. coli and S. cerevisiae $[68,69]$.

Another important challenge in metabolic engineering is satisfying cellular energetic concerns (i.e. thermodyn-amic constraints). For the production of bulk chemicals and biofuels such as ethanol, fermentation is ideal. However, commercial production of 1,3-propanediol has been accom-plished aerobically using engineered E. coli optimized for the redox demands of the corresponding pathway [70]. An important parameter in strain optimization is the balance of energy and cofactor requirements for the metabolic pathways used to generate the target molecule. One example of this is from the production of polyhydroxybu-tyrate (PHB) in E. coli under fermentative conditions. In this study [71], PHB yield was improved by the introduc-tion of UdhA, an NAD-NADP transhydrogenase, to bal-ance NADH and NADPH levels [71]. This work also illustrates the potential of engineered microbes in produ-cing replacements for petroleum-based consumer products other than fuel (see Box 2 for more examples). Supplanting high-value petroleum products, such as plastics, with renewable products is an important first step towards creating sustainable sources for the petroleum products upon which we have come to rely in our daily life. With continued improvements in metabolic engineering, we expect microbial production of bulk commodities to become economically competitive with petrochemicals.

Box 2. Microbial sources of other petrochemical commodities

The petrochemical industry is a source of a variety of other commodities in addition to fuel, Industrial production using engineered microbes also has the potential to replace other crude-oil-derived products [14,84]. Historically, Clostridium strains have been used for the production of a variety of biosolvents, such as acetone, butanol and ethanol [21]. More recently, E. coli strains have been engineered by Dupont/Genencor for the industrial production of 1,3-propanediol from glycerol through an aerobic process [70]. Microbial production of both biodegradable plastics (e.g. PHA (polyhydroxyalkanoate), polylactic acid $[85,86]$ ) and non-biodegrad-able plastics (e.g. polythioesters [87]) provides a range of biobased polymers and represents an emerging field. For example, Metabolix Inc (Cambridge, MA) has commercialized a cost-effective method to produce a PHA-based plastic (Mirel ${ }^{\mathrm{TM}}$ ) through the fermentation of corn sugar [88]. Similarly, adipic acid, which serves as a precursor in nylon production, has been produced with the use of an engineered strain of E. coli [89].

To date, the optimization of bioethanol production has involved considerable metabolic engineering, including the use of efficient alternate routes and enzymes from multiple and often non-model organisms. One such widely used strategy is the high-efficiency PET cassette derived from Z. mobilis [14]. For success in the production of alternative biofuels, similar metabolic engineering strategies will be necessary; for example, the development of targeted and efficient transport systems, the improvement of the resistance of biofuels producers to the toxic effects of accumulating biomolecules, the optimization of carbon flux to the desired products, and the construction of strains that are robust under industrial process conditions [14,72-74]. Some advances that might have enormous potential in the field of microbial biofuel production include the engineer-ing of a reduced-genome E. coli strain that can be used for the systematic design of desired phenotypes [75], and the inter-microbial genome transplantation demonstrated in Mycoplasma caprilocum [76]. These examples represent the first steps towards engineering an entire biological system from the ground up. Massive sequencing efforts exploring the biodiversity of culturable organisms as well as metagenomes, such as the Sargasso Sea project [77], have generated a wealth of genetic information. These endeavors are providing novel biosynthetic pathway infor-mation that can be used to design optimized systems for every fuel category. The rapid advances seen in the de-velopment of these technologies will almost undoubtedly facilitate the efficient and reliable production of systems for novel biofuels. Ultimately, political 
forces, process economics, engine technology, and supply infrastructure will dictate the widespread acceptability and use of these alternate fuel sources.

\section{REFERENCES}

1 (2006) Bioethanol needs biotech now. Nat. Biotechnol. 24, 725

2 Herrera, S. (2006) Bonkers about biofuels. Nat. Biotechnol. 24, 755-760

3 Energy Information Administration (2007) International Energy Outlook 2007, US Department of Energy ( http://www.eia.doe.gov/ oiaf/ieo/pdf/0484.(2007).pdf).

4 Schubert, C. (2006) Can biofuels finally take center stage? Nat. Biotechnol. 24, 777-784

5 Schmidt, L.D. and Dauenhauer, P.J. (2007) Chemical engineering: hybrid routes to biofuels. Nature 447, 914-915

6 Galbe, M. and Zacchi, G. (2007) Pretreatment of lignocellulosic materials for efficient bioethanol production. Adv. Biochem. Eng. Biotechnol. 108, 41-65

7 Merino, S.T. and Cherry, J. (2007) Progress and challenges in enzyme development for biomass utilization. Adv. Biochem. Eng. Biotechnol. 108, 95-120

8 Hahn-Hagerdal, B. et al. (2001) Metablic engineering of Saccharomyces cerevisiae for xylose utilization. Adv. Biochem. Eng. Biotechnol. 73, 53-84

9 Kuyper, M. et al. (2005) Evolutionary engineering of mixed-sugar utilization by a xylose-fermenting Saccharomyces cerevisiae strain. FEMS Yeast Res. 5, 925-934

10 van Maris, A.J. et al. (2007) Development of efficient xylose fermentation in Saccharomyces cerevisiae: xylose isomerase as a key component. Adv. Biochem. Eng. Biotechnol. 108, 179-204

11 Mohagheghi, A. et al. (2002) Cofermentation of glucose, xylose, and arabinose by genomic DNA-integrated xylose/arabinose fermenting strain of Zymomonas mobilis AX101. Appl. Biochem. Biotechnol. 98-100, 885-898

12 Dien, B.S. et al. (2003) Bacteria engineered for fuel ethanol production: current status. Appl. Microbiol. Biotechnol. 63, 58266

13 van Maris, A.J. et al. (2006) Alcoholic fermentation of carbon sources in biomass hydrolysates by Saccharomyces cerevisiae: current status. Antonie Van Leeuwenhoek 90,391-418

14 Jarboe, L.R. et al. (2007) Development of ethanologenic bacteria. Adv. Biochem. Eng. Biotechnol. 108, 237-261

15 Ingram, L.O. et al. (1987) Genetic engineering of ethanol production in Escherichia coli. Appl. Environ. Microbiol. 53, 2420-2425

16 Service, R.F. (2007) Cellulosic ethanol: biofuel researchers prepare to reap a new harvest. Science 315, 1488-1491

$17 \mathrm{Kim}$, Y. et al. (2007) Construction of an Escherichia coli K-12 mutant for homoethanologenic fermentation of glucose or xylose without foreign genes. Appl. Environ. Microbiol. 73, 1766-1771

18 Yoshida, A. et al. (2006) Enhanced hydrogen production from glucose using ldh- and frd-inactivated Escherichia coli strains. Appl. Microbiol. Biotechnol. 73, 67-72

19 Prince, R.C. and Kheshgi, H.S. (2005) The photobiological production of hydrogen: potential efficiency and effectiveness as a renewable fuel.Crit. Rev. Microbiol. 31, 19-31

20 Ezeji, T.C. et al. (2007) Bioproduction of butanol from biomass: from genes to bioreactors. Curr. Opin. Biotechnol. 18, 220-227

21 Rogers, P. et al. (2006) Organic acid and solvent production. In The Prokaryotes (Dworkin, M., ed.), pp. 511-755, Springer

22 Ezeji, T.C. et al. (2004) Butanol fermentation research: upstream and downstream manipulations. Chem. Rec. 4, 305-314

23 Ezeji, T.C. et al. (2005) Continuous butanol fermentation and feed starch retrogradation: butanol fermentation sustainability using Clostridium beijerinckii BA101. J. Biotechnol. 115, 179-187

24 Fukuda, H. et al. (2001) Biodiesel fuel production by transesterification of oils. J. Biosci. Bioeng. 92, 405-416

25 White, S.W. et al. (2005) The structural biology of type II fatty acid biosynthesis. Annu. Rev. Biochem. 74, 791-831

26 Chisti, Y. (2007) Biodiesel from microalgae. Biotechnol. Adv. 25, 294-306

27 Banerjee, A. et al. (2002) Botryococcus braunii: a renewable source of hydrocarbons and other chemicals. Crit. Rev. Biotechnol. 22, 245-279

28 Metzger, P. and Largeau, C. (2005) Botryococcus braunii: a rich source for hydrocarbons and related ether lipids. Appl. Microbiol. Biotechnol. 66, 486-496

29 Park, M.O. (2005) New pathway for long-chain n-alkane synthesis via 1-alcohol in Vibrio furnissii M1. J. Bacteriol. 187, $1426-1429$

30 Wackett, L.P. et al. (2007) Genomic and biochemical studies demonstrating the absence of an alkaneproducing phenotype in Vibrio furnissii M1. Appl. Environ. Microbiol. 73, 7192-7198

31 Cheesbrough, T.M. and Kolattukudy, P.E. (1984) Alkane biosynthesis by decarbonylation of aldehydes catalyzed by a particulate preparation from Pisum sativum. Proc. Natl. Acad. Sci. U. S. A. 81, 6613-6617

32 Dennis, M. and Kolattukudy, P.E. (1992) Acobalt-porphyrin enzyme converts a fatty aldehyde to a hydrocarbon and CO. Proc. Natl. Acad. Sci. U. S. A. 89, 5306-5310

33 Voelker, T.A. and Davies, H.M. (1994) Alteration of the specificity and regulation of fatty acid synthesis of Escherichia coli by expression of a plant medium-chain acyl-acyl carrier protein thioesterase. J. Bacteriol. 176, 7320-7327

34 Campbell, J.W. et al. (2003) A new Escherichia coli metabolic competency: growth on fatty acids by a novel anaerobic beta- oxidation pathway. Mol. Microbiol. 47, 793-805. 
35 Michinaka, Y. et al. (2003) Extracellular secretion of free fatty acids by disruption of a fatty acyl-CoA synthetase gene in Saccharomyces cerevisiae. J. Biosci. Bioeng. 95, 435-440

36 Mullner, H. and Daum, G. (2004) Dynamics of neutral lipid storage in yeast. Acta Biochim. Pol. 51, 323-347

37 Kalscheuer, R. et al. (2006) Neutral lipid biosynthesis in engineered Escherichia coli: jojoba oil-like wax esters and fatty acid butyl esters. Appl. Environ. Microbiol. 72, 1373-1379

38 Holtzapple, E. and Schmidt-Dannert, C. (2007) Biosynthesis of isoprenoid wax ester in Marinobacterhydrocarbonoclasticus

DSM 8798; identification and characterization of isoprenoid coenzyme A synthetase and wax ester synthases. J. Bacteriol. 189, 3804-3812

39 Kalscheuer, R. et al. (2006) Microdiesel: Escherichia coli engineered for fuel production. Microbiology 152, 2529-2536

40 Kuzuyama, T. (2002) Mevalonate and nonmevalonate pathways for the biosynthesis of isoprene units. Biosci. Biotechnol. Biochem. 66, 1619-1627

41 Wake, L.V. and Hillen, L.W. (1980) Study of a 'bloom' of the oil-rich alga Botryococcus braunii in the Darwin river reservior. Biotechnol. Bioeng. 22, 1637-1656

42 Hull, A. et al. (2006) An alternative fuel for spark ignition engines. Int. J. Eng. Res. 7, 203-214

43 Tsukahara, K. and Sawayama, S. (2005) Liquid fuel production using microalgae. J. Jpn. Pet. Inst. 48, 251-259

44 Klein-Marcuschamer, D. et al. (2007) Engineering microbial cell factories for biosynthesis of isoprenoid molecules: beyond lycopene. Trends Biotechnol. 25, 417-424

45 Withers, S.T. and Keasling, J.D. (2007) Biosynthesis and engineering of isoprenoid small molecules. Appl. Microbiol. Biotechnol. 73, 980-990

46 Chang, M.C.Y. and Keasling, J.D. (2006) Production of isoprenoid pharmaceuticals by engineered microbes. Nat. Chem. Biol. 2, 674-681

47 Jin, Y-S. and Stephanopoulos, G. (2007) Multi-dimensional gene target search for improving lycopene biosynthesis in Escherichia coli. Metab. Eng. 9, 337-347

48 Alper, H. et al. (2005) Identifying gene targets for the metabolic engineering of lycopene biosynthesis in Escherichia coli. Metab. Eng. 7, 155-164

49 Martin, V.J. et al. (2003) Engineering a mevalonate pathway in Escherichia coli for production of terpenoids. Nat. Biotechnol. 21, 796-802

50 Ro, D.K. et al. (2006) Production of the antimalarial drug precursor artemisinic acid in engineered yeast. Nature 440,940 943

51 Yoon, S.H. et al. (2007) Increased b-carotene production in recombinant Escherichia coli harboring an engineered isoprenoid precursor pathway with mevalonate addition. Biotechnol. Prog. 23, 599-605

52 Lee, P.C. et al. (2002) Metabolic engineering towards biotechnological production of carotenoids in microorganisms. Appl. Microbiol. Biotechnol. 60,1-11

53 Chang, M.C.Y. et al. (2007) Engineering Escherichia coli for production of functionalized terpenoids using plant P450s. Nat. Chem. Biol. 3, 274-277

54 Donald, K.A. et al. (1997) Effects of overproduction of the catalytic domain of 3-hydroxy-3-methylglutaryl coenzyme A reductase on squalene synthesis in Saccharomyces cerevisiae. Appl. Environ. Microbiol. 63, 33413344

55 Shiba, Y. et al. (2007) Engineering of the pyruvate dehydrogenase bypass in Saccharomyces cerevisiae for high-level production of isoprenoids. Metab. Eng. 9, 160-168

56 Pitera, D.J. et al. (2007) Balancing a heterologous mevalonate pathway for improved isoprenoid production in Escherichia coli. Metab. Eng. 9, 193-207

57 Mijts, B.N. et al. (2004) Engineered carotenoid biosynthetic pathways. Methods Enzymol. 388, 315-329

58 Verwaal, R. et al. (2007) High-level production of beta-carotene in Saccharomycescerevisiaeby successive transformation with carotenogenic genes from Xanthophyllomyces dendrorhous. Appl.

Environ. Microbiol. 73, 4342-4350

59 Vadali, R.V. et al. (2005) Enhanced lycopene productivity by manipulation of carbon flow to isopentenyl diphosphate in Escherichia coli. Biotechnol. Prog. 21, 1558-1561

60 Seo, J.S. et al. (2005) The genome sequence of the ethanologenic bacterium Zymomonas mobilis ZM4. Nat. Biotechnol. 23, 63-68

61 Withers, S.T. et al. (2007) Identification of isopentenol biosynthetic genes from Bacillus subtilis by a screening method based on isoprenoid precursor toxicity. Appl. Environ. Microbiol. 73, 6277-6283

62 Horton, C.E. et al. (2003) Heterologous expression of the Saccharomycescerevisiaealcohol acetyltransferase genes in Clostridium acetobutylicum and Escherichia coli for the production of isoamyl acetate. J. Ind. Microbiol. Biotechnol. 30, 427-432

63 Singh, R. et al. (2008) Aerobic production of isoamyl acetate by overexpression of the yeast alcohol acetyltransferases AFT1 and AFT2 in Escherichia coli and using low-cost fermentation ingredients. Bioprocess Biosys. Eng 31, 299-306

64 Yoshikuni, Y. et al. (2006) Designed divergent evolution of enzyme function. Nature 440, 1078-1082

65 Martin, V.J.J. et al. (2001) The in vivo synthesis of plant sesquiterpenes by Escherichia coli. Biotechnol. Bioeng. 75, 497- 
503

66 Reiling, K.K. et al. (2004) Mono and diterpene production in Escherichia coli. Biotechnol. Bioeng. 87, 200212

67 Tilman, D. et al. (2006) Carbon-negative biofuels from low-input high- diversity grassland biomass. Science 314, 15981600

68 Alper, H. and Stephanopoulos, G. (2007) Global transcription machinery engineering: a new approach for improving cellular phenotype. Metab. Eng. 9, 258-267

69 Alper, H. et al. (2006) Engineering yeast transcription machinery for improved ethanol tolerance and production. Science $314,1565-1568$

70 Nakamura, C.E. and Whited, G.M. (2003) Metabolic engineering for the microbial production of 1,3-propanediol. Curr. Opin. Biotechnol. 14, 454-459

71 Sanchez, A.M. et al. (2006) Effect of overexpression of a soluble pyridine nucleotide transhydrogenase (UdhA) on the production of poly(3-hydroxybutyrate) in Escherichia coli. Biotechnol. Prog. 22, 420-425

72 Chotani, G. et al. (2000) The commercial production of chemicals using pathway engineering. Biochim. Biophys. Acta 1543, 434-455

73 Sardessai, Y. and Bhosle, S. (2002) Tolerance of bacteria to organic solvents. Res. Microbiol. 153, 263-268

74 Koffas, M. and Stephanopoulos, G. (2005) Strain improvement by metabolic engineering: lysine production as a case study for systems biology. Curr. Opin. Biotechnol. 16, 361-366

75 Posfai, G. et al. (2006) Emergent properties of reduced-genome Escherichia coli. Science 312, 1044-1046

76 Lartigue, C. et al. (2007) Genome transplantation in bacteria: changing one species to another. Science 317, 632-638

77 Venter, J.C. et al. (2004) Environmental genome shotgun sequencing of the Sargasso Sea. Science 304, 66-74

78 Sawyer, R.F. (1993) Trends in auto emissions and gasoline composition. Environ. Health Perspect. 101 (Suppl.), 5-12

79 Ghosh, P. et al. (2006) Development of a detailed gasoline composition-based octane model. Ind. Eng. Chem. Res. 45, 337-345

80 Ghosh, P. and Jaffe, S.B. (2005) Detailed composition-based model for predicting the cetane number of diesel fuels. Ind. Eng. Chem. Res. 45, 346-351

81 Hill, J. et al. (2006) Environmental, economic, and energetic costs and benefits of biodiesel and ethanol biofuels. Proc. Natl. Acad. Sci. U. S. A. 103, 11206-11210

82 Granda, C.B. et al. (2007) Sustainable liquid biofuels and their environmental impact. Environ. Prog. 26, 233-250

83 Somerville, C. (2007) Biofuels. Curr. Biol. 17, R115-R119

84 Tyo, K.E. et al. (2007) Expanding the metabolic engineering toolbox: more options to engineer cells. Trends Biotechnol. 25, 132-137

85 Flieger, M. et al. (2003) Biodegradable plastics from renewable sources. Folia Microbiol. (Praha) 48, 27-44

86 Thomsen, M.H. (2005) Complex media from processing of agricultural crops for microbial fermentation. Appl. Microbiol. Biotechnol. 68, 598-606

87 Steinbuchel, A. (2005) Non-biodegradable biopolymers from renewable resources: perspectives and impacts. Curr. Opin. Biotechnol. 16, 607-613

88 Peoples, O.P. et al. (1994) Massachusetts Institute of Technology and Metabolix Inc. Overproduction and purification of soluble PHA synthase, US Patent \#5480794

89 Frost, J.W. and Draths, K.M. (1996) Purdue Research Foundation. Synthesis of adipic acid from biomass-derived carbon sources, US Patent \#5487987

This work was supported by the Director, Office of Science, Office of Basic Energy Sciences, of the U.S. Department of Energy under Contract No. DE-AC02-05CH11231.

This work was supported by the Assistant Secretary for Energy Efficiency and Renewable Energy, Office of Building Technology, State, and Community Programs, of the U.S. Department of Energy under Contract No. DEAC02-05CH11231. 